\title{
HDAC Inhibitors as Anticancer Therapeutics
}

\author{
Sidra Shafique* \\ Department of Reproduction and Developmental Sciences DBMS, Queen's University Kingston, Canada
}

*Corresponding author: Sidra Shafique, Reproduction and Developmental Sciences DBMS, Queen's University Kingston, Canada.
Received Date: September 13, 2018

Published Date: October 04, 2018

\begin{abstract}
Underlying mechanisms of carcinogenic aberrations in genome stems from genetic mutations and epigenetic modulations followed by a cascade of mechanistic events in signaling pathways. Acetylation status have been identified as one of the key markers of cancerous cells along with the over expression of Histone deacetylases (HDACs). In this context, HDACs are the focus of cancer research both from the cause and the treatment perspectives. HDAC inhibitors are one of the time-tested therapeutics and have recently been used as an effective adjuvant with the combination therapeutic regimens of cancers such as breast cancer. Here, we discuss the HDAC inhibitors as cancer treatment option in context with carcinogenic role of epigenetic modifications.
\end{abstract}

\section{Epigenetics and Cancer Development}

Epigenetics include the modifications that may influence the gene expression without directly inducing any changes in the genetic code itself. Genomic structure consists of double helix DNA coiled around nucleosomes in a compact manner. Nucleosomes consist of pairs of four histones including H2A, H2B, $\mathrm{H} 3$ and $\mathrm{H} 4$ while $\mathrm{H} 1$ is the connecting histone. 146 base pairs of DNA are organized around one nucleosome whereas the histone fold domains depend upon the interactions between histones themselves in addition to between histones and DNA double helix. Amino-terminal tails of histones extend out helping to maintain the DNA super helix structure. The acetylation and methylation are the most studied histone modifications in addition to many others recently discovered. Acetylation of histones plays an important role in chromatin remodeling [1].

Histone acetyltransferase (HATs) enzymes relax the DNA coiled around nucleosomes and the transcription factors are able to access the gene promoters. Histone deacetyl transferases (HDACs) are the enzymes that remove the acetyl groups from the histone tails thus change the DNA configuration towards more compact state and decreasing the accessibility of transcription factors resulting in decrease in transcription. The switching between acetylation and deacetylation which is reflected in transcription regulation is the central dogma in cellular differentiation.

The cellular differentiation is the key step during embryonic development as well as cancerous change in dividing cells. HATs result in differentiation while HDACs over-expression results in non-differentiated state of cells [2].
Methylation status as an epigenetic modification plays an important role in cancer development. $\mathrm{CpG}$ islands in the genome consist of DNA regions where Guanine nucleotide is preceded by the Cytosine nucleotide while separated by a phosphate group. Hypo and hyper-methylation status of promoter regions and CpG islands act as on and off switch for gene expression and gene silencing. Whether over-expression of proto-oncogenes or silencing of tumor-suppressor genes, both may result in cancerous aberration in actively dividing cell system and produce the first cancer cell that refuses to die by apoptosis in spite of being abnormal. For example, about $659 \mathrm{CpG}$ islands studied by microarray approach have shown significant gain or loss in ovarian cancer [3,4]. H4 modification has been identified as a hallmark of cancer cells. More specifically, loss of acetylation at the Lys16 in association with tri-methylation loss of Lys20 residues is evidenced in association with the hypomethylatin of repetitive DNA sequences in tumor cells [5].

Cancer cell population bears a lot of heterogeneity resulting in the distinct cell populations in same cancer cell mass. Cancer stem cells (CSC) are identified as one of these sub-populations that have unique characteristics depending upon and resulting from the tumor microenvironment. Targeting the stem cells as a treatment strategy is expected to be successful in context with the knowledge of the causative epigenetic modifications resulting in the production of these cells. The morphology of the CSC depends on the expression of specific surface markers. These cell markers let the plasticity of the cancer cells allowing them being differentiated and de-differentiated back and forth into cancer stem cells, 
progenitor-like cells and differentiated tumor cells. The two popular proposed models of Hierarchic CSC and Dynamic CSC models may largely determine the therapeutic response to cancer treatment and recurrence that in turn is largely directed by the epigenetic coding of these cells. Breast cancer studies have been proto-type to study the cancer stem cell identification, such as CD44+/CD24-, ALDH+ circulating stem cells phenotype play an important role in breast cancer metastasis [6]. Another example of epigenetic role in CSC is depressed immune response by immune system. Immune response to cancer cells by $\mathrm{T}$ cells occurs through the antigen processing genes (TAP). Epigenetic dysregulations in cancer stem cells have been shown by the downregulated expression of TAP genes due to DNA hypermethylation indicating the epigenetic modifications being a candidate niche for therapeutic targets and drug development [7].

\section{HDACi as Prospective Cancer Therapeutics}

HDACs are shown to be over-expressed in various human tumors. HDAC I, II and III in gastric, breast and colorectal cancer. Therefore, HDACs have been studied as biomarkers of tumors to identify the normal tissue and also as the prognostic indicators such as prostate cancer [8]. HDACs are over-expressed in cancer cells and are considered to play a key role in cancer cells survival. HDAC 1, 2, 3 and 6 knock down induce cell cycle arrest and apoptosis in lung, breast and colon carcinomas thus advocating HDACi as promising cancer therapeutics [2].

HDAC classes include I, II, III and IV. HDACs in class III have NAD-dependent catalytic sites and have the overlapping functions with classical HDACs [2]. (West \& Johnstone, 2014). HDAC downregulation and inhibition by small molecules such as SAHA, TSA and Valproic acid produce hyperacetylation of genome, for example HDAC3 deletion results in increased H3K9, K14ac; H4K5ac; and H4K12ac $[9,10]$.

HDACi results in hyperacetylation which results in induction of apoptosis-inducing genes such as encoding the proapoptotic BMF indicating the interaction between apoptotic signaling pathways and the genetic modification. BMF is shown to play a central role in HDAC-mediated apoptosis [11].

The mechanistics of HDACi resulting in biological processes are not fully understood and largely depends on genetic signature of the tumor itself. Nevertheless, the most elucidated outcomes include differentiation, growth arrest, apoptosis induction, inhibition of angiogenesis and last but not the least immunogenicity. Around twenty clinically tested HDACi have been documented being effective in hematological malignancies such as multiple myeloma and acute myeloid leukemia. No HDACi has been seen effective as a monotherapy against cancer by now but mostly are used as an adjuvents in combination treatments [2].

HDACi are used as small molecule therapeutics in various diseases including malignancies. Based on the chemical structure, HDACi include hydroxamic acids (TSA (trichostatin A), vorinostat (SAHA), carboxylic acids (valproate, butyrate), aminobenzamides (entinostat), cyclic peptides (romidepsin) epoxyketones (trapoxins), and hybrid molecules. Out of these vorinostat and romidepsin have been approved for clinical use in cutaneous $\mathrm{T}$ cell lymphoma (CTCL), while active research is being on valproic acid and TSA [2].

Vorinostat (SAHA) is a pan HDAC inhibitor that modifies the acetylation status and induces apoptosis [12]. HDAC not only acetylate the histones but also affect the acetylation and thus activation status of non-histone proteins. Therefore, evidence suggests that HDACi are also effective in cancer therapy through this mechanism of action. Thus, SAHA increases the acetylation of heatshock protein 90 (HSP90) in HER2-overexpressing breast cancer cell lines. The acetylated HSP90 is dissociated from HER2 resulting in the degradation of HER2 [13]. Similarly, Valproic acid affects relatively better against HER2-overexpressing breast cancer cells than HER2-negative [14]. HDAC inhibitors have proven relatively successful for the treatment of hematological malignancies as compared to solid tumors. Therefore, these are used as an adjunct with current regimens of chemotherapies.

\section{Conclusion}

"Science seems slow and cancer fast" by the pace of epigenetic modifications and resulting heterogeneity in cancer cells and CSCs. Histone code and epigenetic changes are the most widely accepted mechanism of the tumerogenesis, maintenance and the progression of cancer cells regulating the gene expression in heterogenous tumor cell populations. The evidence suggests key role of HDACs in cancer development and progression. One of the limitations of cancer treatment include resistance to the treatment. A wellstudied mechanism of resistance is the expression of anti-apoptotic genes by the cancer epigenome. HDACi induce apoptotic gene expression. Apoptosis is evidenced as a preferential pathway of HDAC inhibitors to induce cell death. Although, HDACi have proven to be effective as a component of combination therapy, their side effects in high doses limit their use as independent therapeutics. Better understanding of epigenetic changes in the cancer stem cells may improve the outcomes by HDACi therapies as these have broad targeting capacity. Targeting the epigenetic aberrations in histone code remains an area of active research in order to reach the goal of developing successful cancer therapy with minimal side effects and maximum efficacy.

\section{Acknowledgement}

None.

\section{Conflict of Interest}

No conflict of interest.

\section{References}

1. Luger K, Mäder AW, Richmond RK, Sargent DF, Richmond TJ (1997) Crystal structure of the nucleosome core particle at $2.8 \AA$ resolution. Nature 389(6648): 251-260.

2. West AC, Johnstone RW (2014) New and emerging HDAC inhibitors for cancer treatment. J Clin Invest 124(1): 30-39.

3. Watts GS, Futscher BW, Holtan N, Degeest K, Domann FE, et al. (2008) DNA methylation changes in ovarian cancer are cumulative with disease progression and identify tumor stage. BMC Medical Genomics 1: 47.

4. Irizarry RA, Ladd-Acosta C, Wen B, Wu Z, Montano C, Onyango P, et al. (2009) The human colon cancer methylome shows similar hypo- and 
hypermethylation at conserved tissue-specific $\mathrm{CpG}$ island shores. Nat Genet 41(2): 178-186.

5. Fraga MF, Ballestar E, Villar-Garea A, Boix-Chornet M, Espada J, et al. (2005) Loss of acetylation at Lys16 and trimethylation at Lys20 of histone $\mathrm{H} 4$ is a common hallmark of human cancer. Nature Genetics 37(4): 391-400.

6. Vermeulen L, de Sousa e Melo F, Richel DJ, Medema JP (2012) The developing cancer stem-cell model: Clinical challenges and opportunities. Lancet Oncology 13(2): e83-89.

7. Sultan M, Vidovic D, Paine AS, Huynh TT, Coyle KM, et al. (2018) Epigenetic Silencing of TAP1 in Aldefluor ${ }^{+}$Breast Cancer Stem Cells Contributes to Their Enhanced Immune Evasion. Stem Cells 36(5): 641654.

8. Weichert W, Röske A, Niesporek S, Noske A, Buckendahl AC, et al. (2008) Class I histone deacetylase expression has independent prognostic impact in human colorectal cancer: Specific role of class I histone deacetylases in vitro and in vivo. Clin Cancer Res 14(6): 1669-1677.

9. Bhaskara S, Knutson SK, Jiang G, Chandrasekharan MB, Wilson AJ, Zheng S, et al. (2010) Hdac3 is essential for the maintenance of chromatin structure and genome stability. Cancer Cell 18(5): 436-447.
10. Heideman MR, Wilting RH, Yanover E, Velds A, de Jong J, et al. (2013) Dosage-dependent tumor suppression by histone deacetylases 1 and 2 through regulation of c-Myc collaborating genes and p53 function. Blood 121(11):2038-2050.

11. Zhang Y, Adachi M, Kawamura R, Imai K (2006) Bmf is a possible mediator in histone deacetylase inhibitors FK228 and CBHA-induced apoptosis. Cell Death Differ 13(1): 129-140.

12. Finnin MS, Donigian JR, Cohen A, Richon VM., Rifkind RA, et al. (1999) Structures of a histone deacetylase homologue bound to the TSA and SAHA inhibitors. Nature 401(6749): 188-193.

13. Bali P, Pranpat M, Swaby R, Fiskus W, Yamaguchi H, et al. (2005) Activity of suberoylanilide hydroxamic Acid against human breast cancer cells with amplification of her-2. Clin Cancer Res 11(17): 6382-6389.

14. Damaskos C, Valsami S, Kontos M, Spartalis E, Kalampokas T, et al. (2017) Histone Deacetylase Inhibitors: An Attractive Therapeutic Strategy Against Breast Cancer. Anticancer Res 37(1): 35-46. 